\title{
Señal respiratoria a partir del acondicionamiento electrónico de la señal ECG
}

\author{
Respiratory signal from the ECG electronic conditioning
}

\author{
James Cowans Rojas, Nancy Olarte López \\ Tecnología en Electrónica y Comunicaciones, Universidad Militar Nueva Granada, Bogotá., Colombia \\ Grupo de investigación e innovación Tecnológica en Electrónica y Comunicaciones - GI-iTEC \\ u1701437@unimilitar.edu.co \\ nancy.olarte@unimilitar.edu.co
}

\begin{abstract}
Resumen- El presente artículo evidencia como se planteó el diseño e implementación de un prototipo para el acondicionamiento de la señal respiratoria a partir del tratamiento de la señal electrofisiológica ECG.

Dentro de la metodología se propuso la implementación de varios esquemas circuitales, manejando dos escenarios importantes, la adquisición de la señal ECG mediante un simulador de paciente, y el tratamiento de la misma por medio de componentes electrónicos no invasivos para obtener la señal de respiración.
\end{abstract}

De manera inicial, se realiza una breve explicación de la señal electrocardiográfica y respiratoria junto con su relación, luego se lleva a cabo una descripción sobre las fases y métodos implementados para la obtención electrofisiológica de ambas señales, posteriormente se realiza un análisis comparativo en amplitud, periodo y frecuencia y por último la discusión indicando ventajas y desventajas del trabajo junto con sus conclusiones, proyectando para posteriores trabajos la telemetría o transmisión a distancia de estas señales acondicionadas.

Palabras clave- Señal ECG, Señal Electrofisiológica, Señal respiratoria, Simulador de paciente.

Abstract- This paper demonstrates how the design and implementation of a prototype design of the respiratory signal from electrophysiological treatment of ECG signal was raised.

Within the methodology implementing various circuital schemes proposed, handling two major stages, the acquisition of the ECG signal through a patient simulator, and processing thereof by means of non-invasive for the respiration signal electronics.

Initial way, a brief explanation of the electrocardiographic and respiratory signal along with their relationship, then carried out a description of the phases and implemented methods for electrophysiological obtaining both signals is performed, then a comparative analysis is performed in amplitude, period and frequency and finally the discussion indicating advantages and disadvantages of working together with its findings, projecting for further work telemetry or remote transmission of these conditioned signals.

Key Word - ECG Signal, Signal Electrophysiological, Patient simulator, Respiratory Signal.

\section{INTRODUCCIÓN}

A través del tiempo, se han tenido amplios desarrollos en el área de procesamiento de señales para la correcta captura, interpretación y medida de los diferentes datos fisiológicos.

Sin embargo aunque existen extensos estudios, diseños y desarrollos en esta área y en el almacenamiento de datos, actualmente se tienen inconvenientes como lo son las técnicas utilizadas para capturarlas, en lo posible mediante mecanismos no invasivos, la interpretación de los datos en pantalla sin perturbación de la señal, los parámetros de seguridad de la información de los pacientes y normatividad a implementar.

Los dispositivos implantados para controlar el estado de los pacientes como por ejemplo la oximetría, ritmo cardiaco, presión ventricular, entre otros, han evolucionado para su interacción con el paciente, pero más que eso, han evolucionado para la transmisión de los datos registrados teniendo un eficiente rendimiento en la supervisión y seguimiento de las condiciones del paciente en tiempo real [1]. 
Es importante que, cuando se trata de sensores, para adquirir distintos tipos de señales, se consideren dos parámetros fundamentales: ser en lo posible no invasivos y por otro lado, que los datos fisiológicos a adquirir sean procesados para convertirlos en señales digitales para su posterior análisis [2]. También, han existido sistemas para la experimentación y tratamiento de señales en los animales, para esto se han venido utilizando transductores convirtiendo señales eléctricas en voltaje; un ejemplo claro de un transductor es el electroquímico o electrodo, empleados para medir potenciales en el electrocardiograma, electroencefalograma y electromiograma [3].

Pero aun así hoy en día surgen inconvenientes como dónde y cuándo debe usarse este tipo de información, la definición de protocolos y el uso de la normatividad adecuada tanto para los médicos como la parte técnica del sistema remoto de consulta [4].

Éste proyecto de iniciación científica (PIC-ING-2148. Análisis de la Frecuencia respiratoria a partir de la Frecuencia Cardíaca mediante el acondicionamiento electrónico de la señal ECG), del Grupo de Investigación e innovación Tecnológica en Electrónica y Comunicaciones (GI-iTEC), semillero Faraday del programa de la Tecnología en Electrónica y Comunicaciones de la Universidad Militar Nueva Granada.

\section{REFERENTE TEÓRICO}

Una señal electrofisiológica es aquella que proviene de la actividad eléctrica de órganos o fibras musculares, adquirida mediante electrodos o sensores de superficie, y es de gran interés en diagnóstico clínico [5]. Un tipo específico de este tipo de señal electrocardiográfica o ECG, adquirida mediante técnicas no invasivas con sensores de pulso.

\section{Señal Electrocardiográfica o ECG}

Una de las señales biológicas más importantes estudiada y documentada ampliamente por numerosos investigadores en el campo de la bioingeniería es la electrocardiográfica (ECG), onda que muestra el comportamiento o activación eléctrica del corazón, y que se obtiene a través de un electrocardiógrafo, el cual registra dicho comportamiento a través de electrodos conectados al cuerpo de un paciente midiendo la diferencia de potencial eléctrico producido en estos puntos.
Los cambios en los patrones de la señal ECG pueden ocurrir por condiciones en el que por ejemplo: el corazón se agranda, trastornos de conducción o enfermedades valvulares [6]. Los intervalos ocurridos entre las deflexiones de esta onda se denominan segmentos y las distancias entre ondas se llaman intervalos, es así como el ECG se divide en varios segmentos e intervalos (Ver Figura 1) y es precisamente el estudio de los mismos en cuanto a tiempos de ocurrencia, forma, intensidad, entre otras características, los que determinan algún tipo de patología en un paciente.

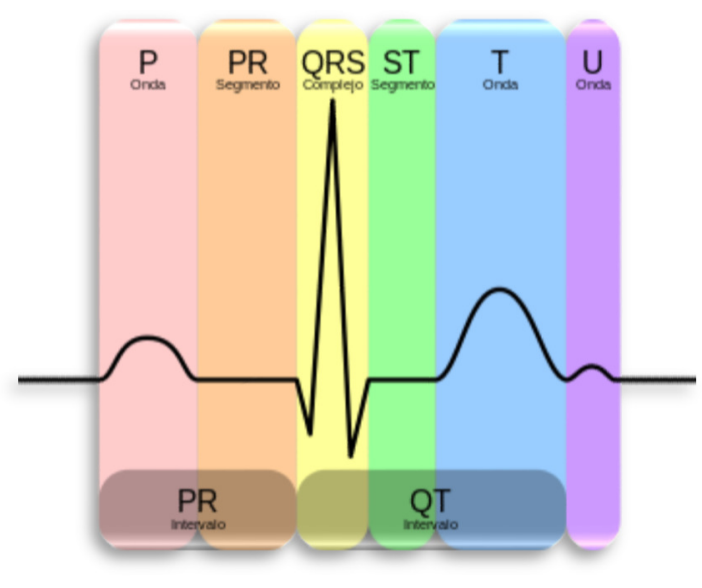

Figura 1. Intervalos Señal ECG [7].

La onda P: indica que las aurículas son estimuladas de forma eléctrica para enviar la sangre hacia los ventrículos. Luego el complejo QRS: el cual indica que los ventrículos son estimulados eléctricamente para bombear la sangre hacia fuera. El segmento ST: indica la cantidad de tiempo que ocurre desde el final de una contracción de los ventrículos hasta el comienzo del periodo de reposo. La onda siguiente se denomina onda $\mathrm{T}$ la cual muestra el periodo de recuperación de los ventrículos. [8]

Ahora bien, para llegar a un diagnóstico evaluando y estudiando una señal ECG de tal manera que dicho diagnóstico sea acertado y confiable se deben cumplir ciertos parámetros en todo el proceso, desde la captura (obtención) de la señal en un paciente hasta la correcta visualización de la misma en algún tipo de dispositivo por parte del especialista.

Por lo anterior son importante los puntos de referencia donde se coloquen los electrodos. Tenerlos en diferentes posiciones, permite apreciar mejor ciertas áreas del corazón.

Para este caso específico de este estudio de investigación, se utilizaran, las derivaciones bipolares, porque estas registran la diferencia de potencial entre dos extremidades del cuerpo. 
Estas se registran de la siguiente forma:

- Brazo izquierdo (LA) y brazo derecho (RA)

- Pierna izquierda (LF) y brazo derecho (RA)

- Pierna izquierda (LF) y brazo izquierdo (LA)

Para lograr medir las derivaciones bipolares es necesario la ubicación adecuada de los electrodos en el cuerpo humano. A este sistema también se le llama triangulo de Einthoven y su distribución se puede apreciar en la siguiente Figura 2.

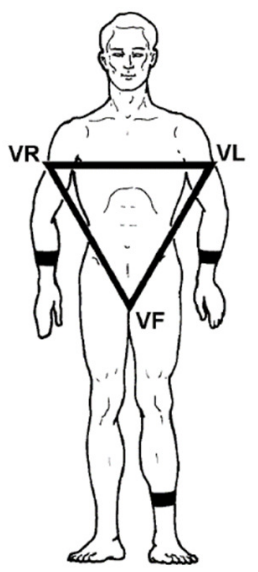

Figura 2. Triángulo de Einthoven [9].

Por otro lado, si se desea enviar dicha señal a un sitio remoto para un diagnóstico a distancia (telediagnóstico) también se debe contar con las técnicas adecuadas para el transporte (seguridad, compresión, etc). Es así como a lo largo de las últimas décadas se han implementado diferentes técnicas en la captura de una señal ECG así como también distintas técnicas para interpretar un electrocardiograma, para lo cual se han desarrollado varios métodos con el tiempo, entre ellos distintos algoritmos y modelos matemáticos, la implementación de redes neuronales, filtros digitales y Transformada Wavelet entre otros [10].

\section{Señal Respiratoria}

Inicialmente la respiración se define como el proceso por el cual los seres vivos absorben y expulsan el aire, [11] debido a esto la señal tiene diferentes características en su forma entre las que se tiene en cuenta el tiempo inspiratorio o de inhalación de aire donde se expande la caja toráxica y el espiratorio en el cual se exhala el aire y se reduce el tamaño de la caja toráxica.

Ambos momentos (inhalación y espiración), permiten ver los tiempos altos y bajos del patrón de onda respiratoria (Ver
Figura 3), para un periodo total de señal de 4.55 segundos, el cual incluye:

Tiempo inspiratorio: 3.20 segundos

Tiempo espiratorio: 1.35 segundos

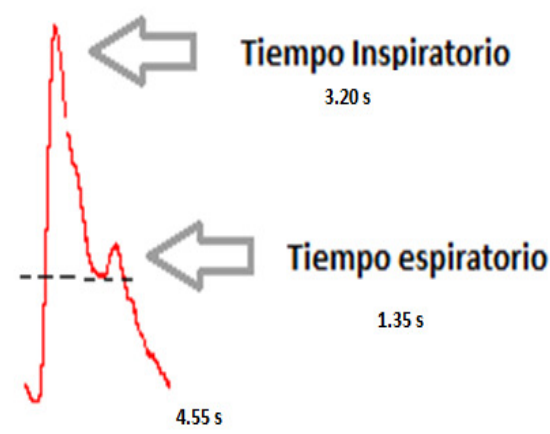

Figura 3. Forma de Onda señal respiratoria. Fuente Autor.

Por otro lado, existen diferentes tipos de anomalías respiratorias algunas de estas relacionadas con la parte cardiaca entre ellas están:

Arritmia sinusal respiratoria: Consiste en la aceleración de la frecuencia cardiaca en la inspiración y su disminución en la espiración. Se observa en un $50 \%$ de adolescentes. [12].

Apnea: La respiración que se hace lenta o se detiene por cualquier causa [13].

Apnea obstructiva: Ausencia hasta del $90 \%$ de la señal respiratoria tiempo de duración hasta 10 segundos [13].

Hipopnea: Reducción significativa de la respiración entre $30 \%$ y $90 \%$ [13].

Taquipnea: Frecuencia respiratoria superior a 20 respiraciones por minuto [14].

Bradipnea: Frecuencia respiratoria inferior a 12 respiraciones por minuto [14].

Disnea: Sensación subjetiva del paciente de dificultad o esfuerzo para respirar [14].

Así mismo, para detectar estas patologías mediante la lectura óptima del registro de la señal respiratoria por medio del especialista, se deben tener en cuenta diferentes aspectos, entre ellos implementar una técnica en lo posible no invasiva para el 
paciente y por supuesto la descripción detallada de su forma de onda sin perturbaciones, para así tratarla, procesarla y analizarla.

\section{Relación entre la señal Electrocardiográfica y Respiración}

Particularmente, uno de los tipos de anomalías respiratorias descritas anteriormente es la Arritmia sinusal respiratoria o $R S A$. Ésta patología relaciona la valoración cíclica de la frecuencia cardiaca asociada con la respiración, es decir que la frecuencia cardiaca se acelera durante la inspiración y disminuye durante la espiración [15].

La magnitud de esta arritmia es independiente en cada individuo y es una anomalía que tiende a disminuir el valor de la frecuencia respiratoria [15].

Por otro lado, la modulación de la amplitud del patrón de onda RS (es decir el máximo pico y mínimo de la señal ECG), se relaciona también con los procesos de inspiración y espiración del proceso de respiración.

Debido a que durante la inspiración el vértice del corazón se amplía hacia el abdomen debido a la expansión de la caja torácica y desplazamiento hacia abajo del diafragma al llenarse de aire los pulmones. Por otro lado, durante la espiración se desplaza el diafragma hacia arriba lo que permite la exhalación de aire comprimiendo el vértice del corazón hacia el pecho, de esta manera se cambia el vector cardiaco eléctrico modulando la amplitud de la señal ECG inducida por la respiración [15].

\section{METODOLOGÍA}

En esta investigación, la metodología se llevó a cabo mediante una serie de fases, las cuales se pueden apreciar a continuación:

- Primera fase: Generación de la señal

- Segunda fase: Acondicionamiento de la señal

- Tercera fase: Filtrado y obtención de la frecuencia respiratoria

\section{A. Primera Fase: Generación de la señal}

En esta fase se tuvo en cuenta el simulador de paciente Fluke PS420 (Ver Figura 4) el cual es de gran importancia ya que genera cuatro tipos de señales: temperatura, presión sanguínea, electrocardiográfica o ECG y respiración.

La señal objeto inicial de este estudio fue la Electrocardiográfica o ECG debido a su profundo análisis y trayectoria en el campo de la bioingeniería en diferentes investigaciones.

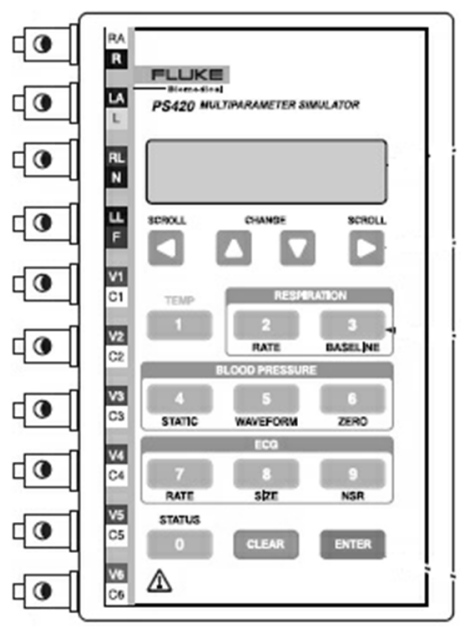

Figura 4. Simulador de paciente FLUKE PS420 [16].

Para que el paciente pueda generar la señal, se necesitan unos cables blindados llamados latiguillos. Estos se conectan a las derivaciones que tiene el paciente en la parte izquierda. Se toman las dos primeras derivaciones que son brazo derecho y brazo izquierdo (RA, LA) y como referencia o polo a tierra, bien sea el pie derecho o el pie izquierdo (RF, LF). Esto se hace para cumplir con las derivaciones estándares del triángulo de Einthoven, para generar la onda cardiaca. Una vez conectados los latiguillos, se procede a ingresar el código correspondiente a la ECG en el simulador de paciente.

\section{B. Segunda Fase: Acondicionamiento de la señal}

En esta fase, luego obtener la señal ECG, se pasa por una serie de etapas: amplificación de la señal, filtrado y finalmente ajuste al nivel DC o en corriente directa, lo anterior para que la señal se encuentre en el orden de 0 a 5 voltios, es decir sin voltajes negativos al ser procesada y visualizada.

Este módulo es de gran importancia, ya que se encarga de interpretar y acondicionar las señales provenientes del paciente, para que posteriormente serán procesadas.

Para la amplificación, se utilizó el amplificador operacional AD620 (Ver Figura 5), el cual ha sido utilizado ampliamente en la cardiografía, por su estabilidad y ganancia, que se encuentra entre 1 y 1000 . Su alimentación varía en el rango de $2,3 \mathrm{~V}$ a $18 \mathrm{~V}$.

También dentro de esta etapa se utilizó el amplificador JFET TL084 por su bajo consumo de potencia y bajo ruido. 


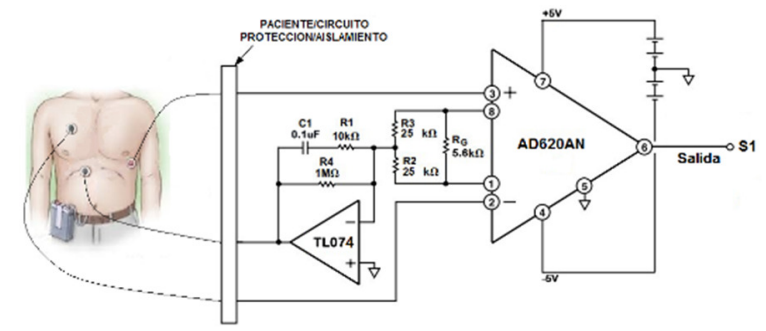

Figura 5. Acondicionamiento y amplificación de la señal

La etapa de filtrado se compone de tres filtros: Filtro pasa bajo, filtro pasa alto y filtro Notch.

El filtro pasa bajo (Ver Figura 6) y pasa alto (Ver Figura 7) limitan el espectro de la frecuencia de la señal ECG entre 0,5 $\mathrm{Hz}$ y $150 \mathrm{~Hz}$.

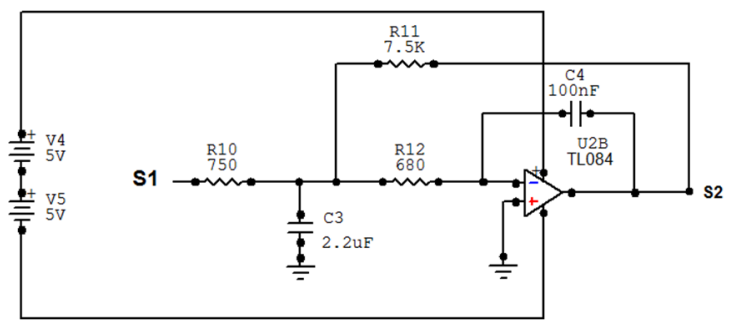

Figura 6. Filtro pasa bajo

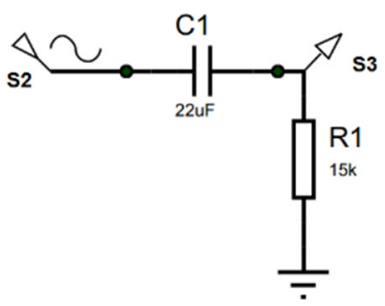

Figura 7. Filtro pasa alto

Y el filtro Notch elimina frecuencias e interferencias de $60 \mathrm{~Hz}$ (Ver Figura 8).

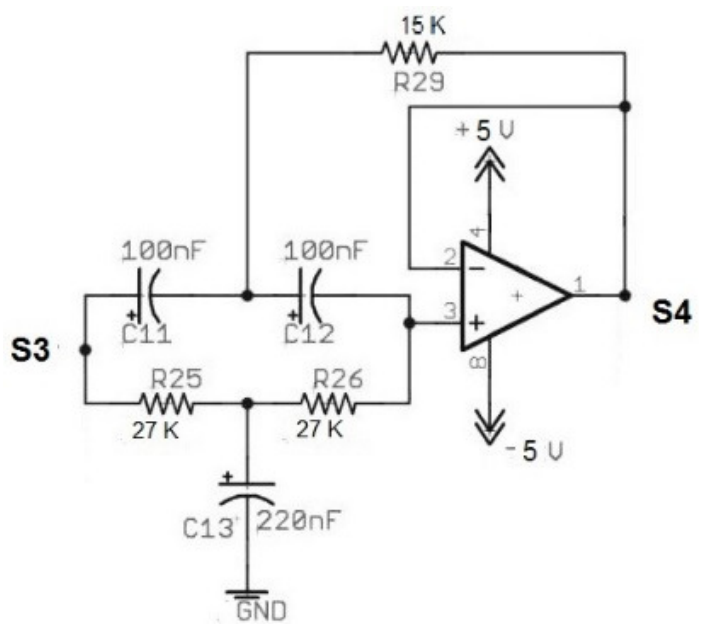

Figura 8. Filtro Notch

Una vez filtrado el ruido de señales indeseadas, se procede a la etapa de amplificación en donde su usa un TL084 con ganancia de 10 (Ver Figura 9).

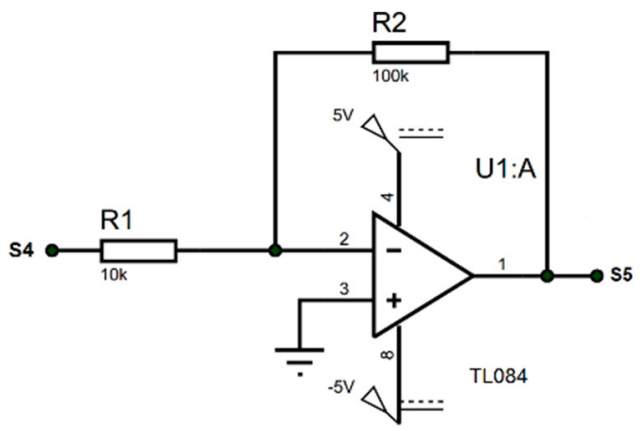

Figura 9. Amplificación señal

Por ultimo un ajuste de nivel DC de la señal (Ver Figura 10), el cual permitirá pasar valores positivos de voltaje o amplitud de la señal.

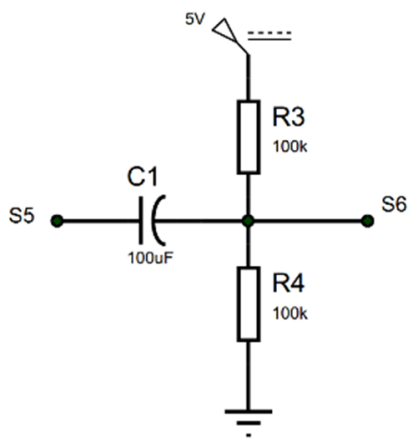

Figura 10. Ajuste nivel DC

C. Filtrado y obtención de la frecuencia respiratoria 
Para esta etapa se tuvo en cuenta que, dentro de los beneficios del simulador del Paciente Fluke 420, se tienen los mismos valores de impedancia para la señal ECG y de respiración tal y como lo muestra la Figura 11 del manual del paciente en sus páginas 4 y 8 .

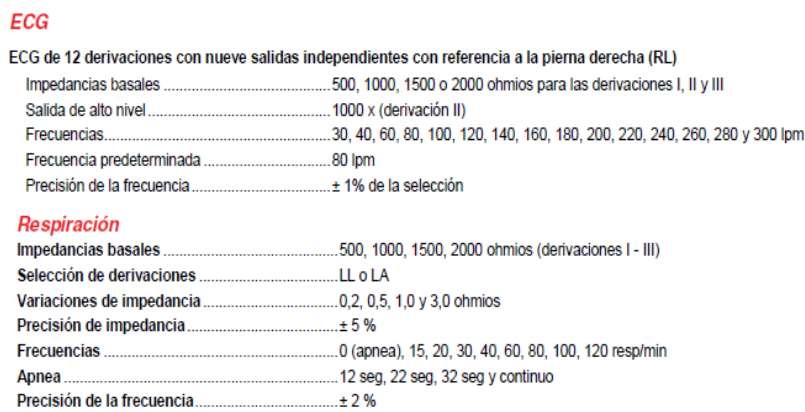

Figura 11. Impedancias basales señales ECG y respiración

Estas impedancias de entrada del equipo son de las derivaciones LL o pierna izquierda o LA brazo izquiero y corresponden a valores de $500 \Omega, 1000 \Omega, 1500 \Omega$ y $2000 \Omega$.

Para el diseño se escoge la impedancia más baja para que los datos a visualizar no se opongan al paso de la corriente.

\section{RESULTADOS}

Luego de realizar las pruebas en protoboard de los diseños de las etapas ya explicadas, a continuación se tienen los resultados.

En la siguiente figura 12 se observa que la señal ECG muestra continuidad en el tiempo pero contiene perturbación en sus niveles de amplitud, por lo anterior fue necesario filtrar el ruido indeseado, adicionando a la salida del circuito el filtro Notch.

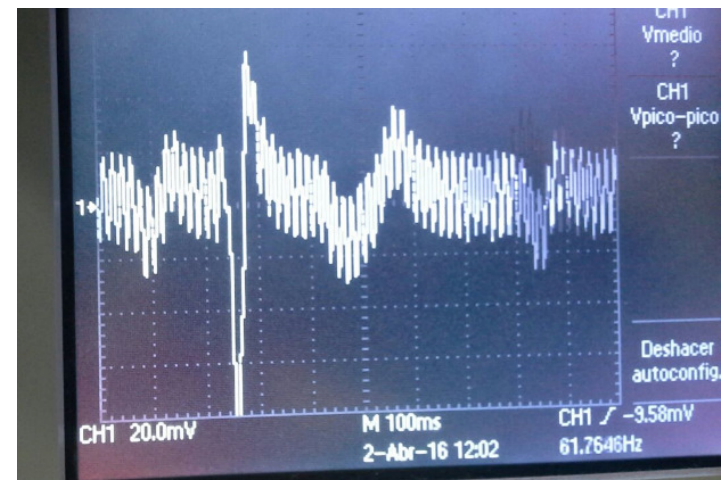

Figura 12. Señal ECG obtenida con perturbaciones
En la Figura 13 se muestra la señal sin ruído y en la Figura 14 se evidencia la continuidad de dos patrones de la señal ECG en el tiempo

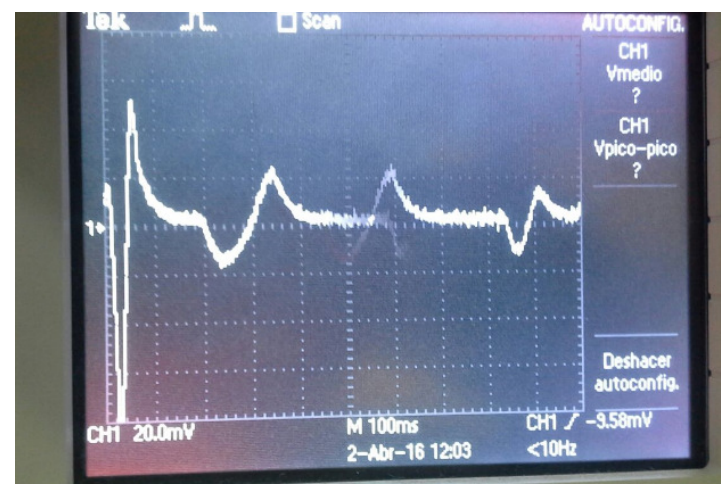

Figura 13. Señal ECG sin ruído

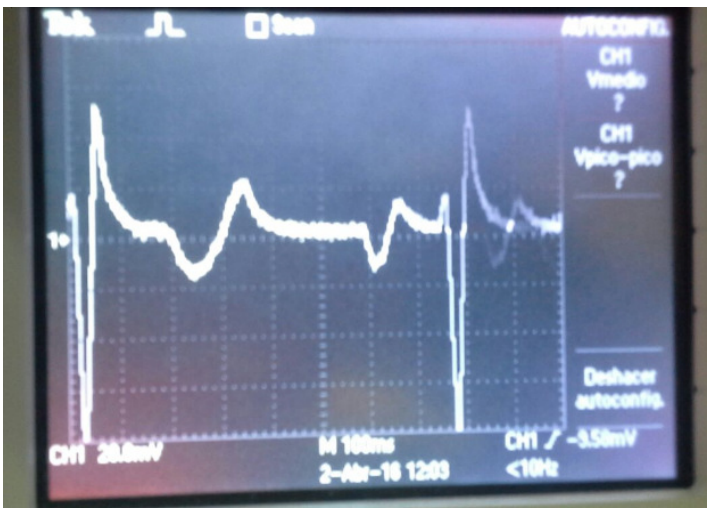

Figura 14. Dos patrones de la señal ECG

Posterior a la prueba inicial, se realiza una nueva prueba con una frecuencia de 60 pulsaciones por minuto o 60ppm donde se visualiza en la pantalla del osciloscopio tres pulsaciones aunque el resultado de esta y la anterior prueba arrojan una señal ECG al revés del patrón usual (Ver Figura 15).

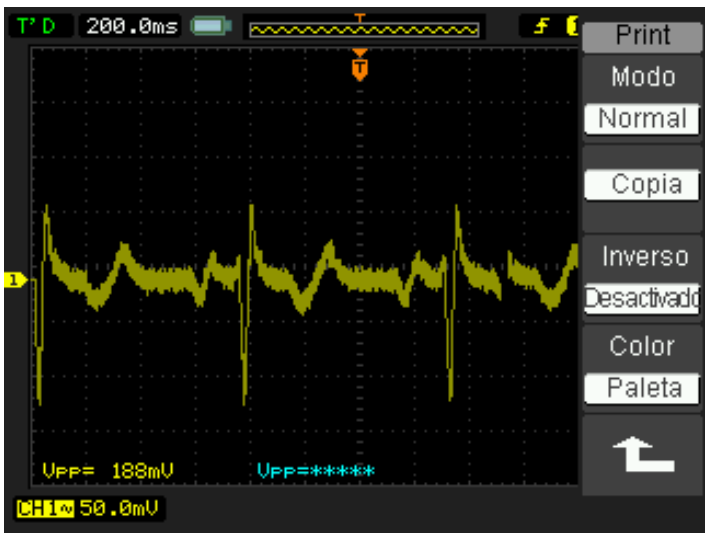

Figura 15. Señal ECG con 60ppm 
Debido a esta respuesta fue necesario incluir como etapa final un inversor TTL 7404, obteniendo la señal que se presenta a continuación en la Figura 16.

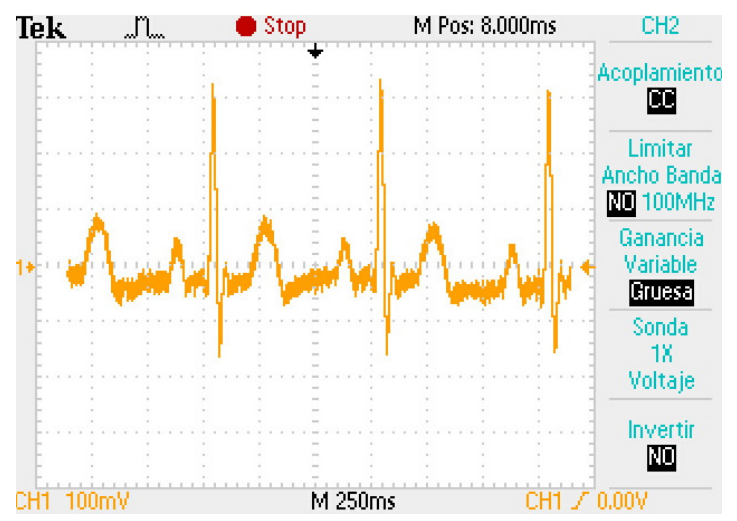

Figura 16. Señal ECG luego del inversor

Utilizando las mismas derivaciones del simulador del paciente para la señal ECG, es decir LL o LA, se cambia el código correspondiente de señal ECG de $60 \mathrm{~Hz}$ (Código 172) al de respiración (Código 153).

Como no era posible comparar ambas señales debido a que se estaban empleando las mismas derivaciones de la señal ECG como etapa complementaria para obtener la señal respiratoria, se compara en un canal adicional la señal resultante de respiración con una señal tipo seno proveniente del generador a una frecuencia de $60 \mathrm{~Hz}$ (Ver Figura 17).

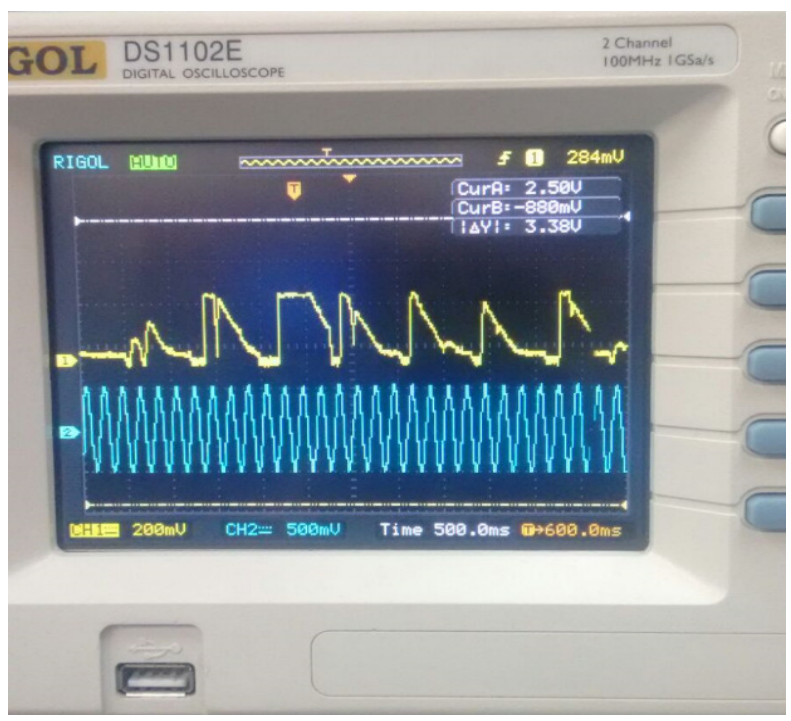

Figura 17. Señal de respiración resultante comparada con señal tipo seno a $60 \mathrm{~Hz}$
Como se puede observar en la figura anterior, la señal resultante respiratoria tiene perturbaciones, lo que hace necesario utilizar como etapa final de nuevo un filtro Notch, cuyo resultado es el de la siguiente Figura 18.

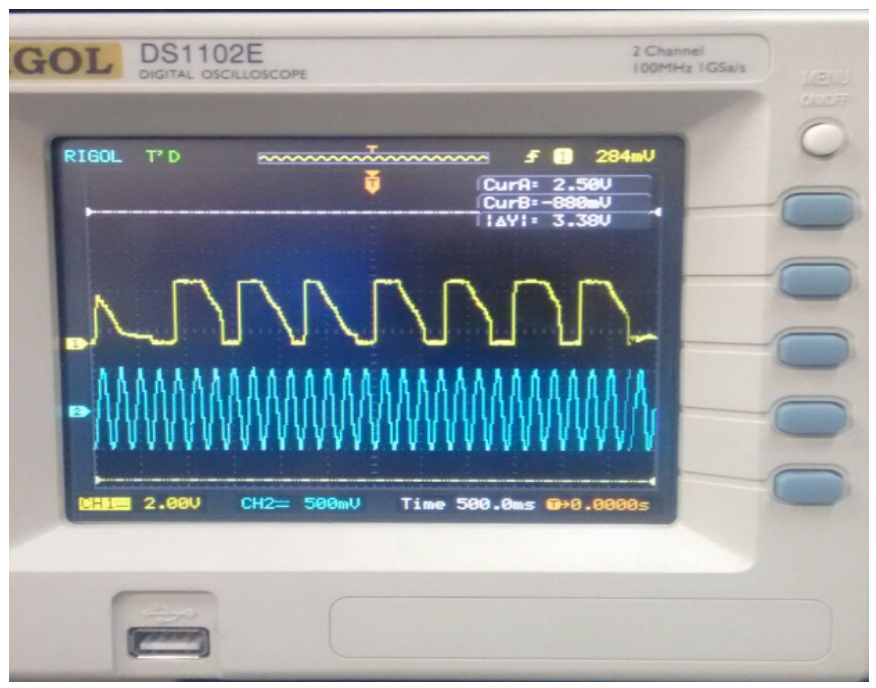

Figura 18. Señal de respiración luego de filtrado

Por lo anterior se deduce que, por cada ciclo completo de señal respiratoria se tienen 4 pulsaciones de la señal ECG, es decir a una frecuencia de $60 \mathrm{~Hz}$ o ppm (pulsaciones por minuto) de señal ECG, se obtienen 15 ciclos completos o $15 \mathrm{~Hz}$ de la señal de respiración.

En otras palabras, cada 4 pulsaciones ECG se obtiene un ciclo completo de señal de respiración.

\section{CONCLUSIONES}

El uso inicial del simulador del paciente fue primordial para los resultados aquí evidenciados, debido a que al obtener la señal en pacientes reales se debe tener los permisos adecuados para realizar las pruebas y con muestra o población real se puede obtener más ruido del deseado debido al contacto de los electrodos con la piel.

Por otro lado, en un paciente real, también se debe tener en cuenta que la medición directa puede ocasionar estrés, ya que pueden llegar a considerar que están bajo un experimento. El estrés ocasionado puede derivar señales imprecisas o alteraciones en el patrón ECG y de respiración, provocando diferentes tipos de frecuencia en ambas señales. 
Se considera a muy corto plazo seguir trabajando en etapas de filtros que mejoren la calidad del patrón de onda de la señal respiratoria.

Por último, perfeccionar la valoración entre la frecuencia cardiaca y la respiratoria aportaría ampliamente en la detección temprana de la Arritmia sinusal respiratoria o RSA y su oportuno tratamiento

\section{RECOMENDACIONES}

Se considera a muy corto plazo seguir trabajando en etapas de filtros que mejoren la calidad del patrón de onda de la señal respiratoria.

Así mismo se considera evaluar con frecuencias superiores a las 60 pulsaciones por minuto o $60 \mathrm{~Hz}$ e incluir el uso de otros simuladores como el SHIELD - EKG - EMG Arduino Electrocardiography EKG Electromiography EMG shield tarjeta que permite el acondicionamiento y visualización de la señal electrocardiográfica o también bajo registros de electrocardiogramas de pacientes reales de la base de datos del MIT (Massachusetts Institute of Technology o Instituto Tecnológico de Massachusetts) los cuales pueden ser procesados bajo el software Matlab.

Para estudios a mediano plazo se busca almacenar los tipos de señales procesadas e incluir para ECG las derivaciones precordiales, además de otro tipo de señales electrofisiológicas que se vienen tratando como son la pletismográfica y oximétrica.

\section{AGRADECIMIENTOS}

A la Universidad Militar Nueva Granada por financiar el Proyecto de Iniciación Científica PIC - ING - 2148. Así mismo al programa de la Tecnología en Electrónica y Comunicaciones y Grupo de Investigación e innovación Tecnológica en Electrónica y Comunicaciones GI-iTEC junto al semillero Faraday por apoyar la investigación formativa en los estudiantes.

\section{REFERENCIAS}

[1]. M. Paredes-Castro y C. Silva-Cárdenas, Diseño e implementación de un instrumento de medición multifrecuencial de impedancia eléctrica de tejidos biológicos.

[2]. A. Jurik, «Biotelemetry and Body Sensors: Enabling ECG Monitoring» 2009.

[3]. Tur, «Instrumentación y métodos de registro en experimentación animal».

[4]. C. H. Salvador, «Modelo de historia clínica electrónica para teleconsulta médica,» 2004.

[5]. Cherniz, A. S., Cian, L. M., \& Escobar, S. (2002). Sistema de adquisición multicanal para el registro de señales electrofisiológicas. Salão de iniciação Científica (14.: 2002: Porto Alegre, RS). Livro de resumos. Porto Alegre: UFRGS, 2002.

[6]. Herrera, «Sistema para la visualización de señales cardiacas,» Revista de Investigaciones Universidad del Quindío, vol. 17, pp. 175-187, 2007.

[7]. http://es.wikipedia.org/wiki/Electrocardiograma\#/me dia/File:EKG_Complex_es.svg.

[8]. Cartaya \& Colorado, "Cardiofile: A Multi-event Recorder for use in Defibrillators", (Book Style), 2008, 2, pp. 25-30.

[9]. Franco, "The Electrocardiogram leads", (Book Style), pp. 14

[10]. C. Michalakopoulos, «A combinatorial model for ecg interpretation» 2009.

[11]. Godoy Diana, Diseño y Construcción de una Plataforma de Telemedicina para el monitoreo de Bioseñales

[12]. Morillo, C. A., \& Guzmán, J. C. (2007). Taquicardia sinusal inapropiada: actualización. Revista Española de Cardiología, 60(Supl. 3), 10-14.

[13]. Trávez Proaño, E. I. (2015). Estrategia educativa para disminuir la incidencia de distres respiratorio en neonatos atendidos en el Hospital José María Velasco Ibarra Tena 2013.

[14]. Meneses A, Toloza D. (2006). Diseño y construcción de un monitor de signos vitales. Dalcame

[15]. Florentin D. Estimación y evaluación de la señal respiratoria a partir de la señal fotopletismográfica de pulso, señal de presión arterial y señal electrocardiográfica. Universidad de Zaragoza. Centro Politécnico Superior. Septiembre de 2010 .

[16]. Manual Fluke PS-420. Tomado de: http://setgad.com/wpcontent/uploads/2015/07/PS420-UM-ESP.pdf 